\title{
Estenosis de manga gástrica. Conversión a by-pass gástrico (video)
}

Gastric sleeve stenosis. Conversion to gastric bypass (video)

Javier Chinelli ${ }^{1}$, Gabriel Massaferro ${ }^{2}$, Rodrigo Hernández ${ }^{3}$, Florencia Sarries ${ }^{4}$, Gustavo Rodríguez Temesio $^{5}$

DOI: $10.31837 /$ cir.urug/3.2.5

Recibido: 20 de junio de 2019

Aceptado: 30 de junio de 2019

\section{Introducción}

La cirugía bariátrica y metabólica es un pilar importante en el tratamiento de la obesidad mórbida, siendo la gastrectomía vertical en manga (o manga gástrica) uno de los procedimientos más frecuentemente realizados, con una baja tasa de complicaciones. Entre ellas se encuentran la fístula, estenosis, kinking,

\section{ESTENOSIS DE MANGA GÁSTRICA}

\section{CONVERSION A BY-PASS GÁSTRICO}

Dres. Javier Chinell, Gabriel Massaferra, Rodrigo Hernández, Florencia Samies, Gustavo Rodriguez.

Clinica Quirúrgica 2 Hospital Maciel twisting, reflujo gastroesofágico y la dilatación. Las estenosis tardías pueden obedecer a varias causas, como la utilización de una bujía demasiado pequeña para calibrar la manga, o errores técnicos al efectuar la sección y grapado, sobre todo al dirigir el primer disparo sobre el antro hacia la el sinusangularis. El twisting puede deberse a la tracción desigual de las caras anterior y posterior al momento de cerrar y disparar la máquina de sutura.

\section{Descripción de contenidos}

Se presenta el caso de una paciente de 52 años, colecistectomizada, a la que se realizó en 2015 una manga gástrica laparoscópica. Dos años después comienza con vómitos postprandiales, por lo que se solicitan fibrogastroscopía y tránsito esofagogastroduodenal que evidencian una estenosis mesogástrica de $3 \mathrm{~cm}$ de longitud y dilatación supraestenótica. Se decide realizar cirugía de revisión por abordaje laparoscópico, constatándose twisting de la manga, por lo que se convierte a by-pass gástrico con técnica simplificada. Se confecciona pouch gástrico, se confecciona asa alimentaria de unos $110 \mathrm{~cm}$, anastomosis gastro-yeyunal al pouch, anastomosis entero-entérica al pie y sección del asa biliar entre ambas anastomosis. De esta forma quedan posicionadas de derecha a izquierda las asas alimentaria, común y biliar respectivamente.

${ }^{1,2,3,4,5}$ Clínica Quirúrgica 2. Hospital Maciel. Facultad de Medicina. Universidad de la República. Montevideo, Uruguay jchinelli01@hotmail.com 